\title{
Validation of fluid milk consumer segments using qualitative multivariate analysis
}

\author{
W. S. Harwood and M. A. Drake* (1) \\ Department of Food, Bioprocessing, and Nutrition Sciences, Southeast Dairy Foods Research Center, North Carolina State University, \\ Raleigh, 27695
}

\begin{abstract}
Consumption of fluid milk in the United States has declined in recent years. To increase appeal and meet ever-changing consumer demands, several product features have been introduced to the fluid milk market. As such, it is imperative to assess consumer sentiments from both quantitative and qualitative perspectives to better understand the effect of various product offerings. The objectives of this study were to identify fluid milk consumer segments that were characterized by preference for specific product features and to verify those sentiments using qualitative multivariate analysis (QMA). An adaptive choice-based conjoint survey $(\mathrm{n}=719)$ was designed to explore consumer desires regarding the fat content, package type, shelf life, label claims, and prices of commercial milks. Part-worth utilities from the conjoint task were subsequently clustered, revealing 4 unique consumer segments. Representative consumers from each cluster $(\mathrm{n}=18$ total) were selected to participate in a 4-wk QMA study, consisting of a homeusage test followed by focus groups and projective mapping. Nine commercial milks representing various pasteurization methods, label claims, and packaging types were used within the study. When analyzed by segment, significant differences in conjoint utilities for specific product features were identified. Overall, price was the largest differentiator of segments, followed by fat content, shelf life, and milk designation. Several of the segmental differences were confirmed in the QMA study, with sentiments regarding price and milk type (i.e., conventional, organic, local) consistent between conjoint survey and QMA for each consumer group. Within the projective mapping exercise, intrinsic sensory properties, price, and quality perception were the primary differentiators for fluid milks. Comments from QMA journal entries and focus groups revealed that consumer segments with preferences for noncon-
\end{abstract}

Received October 26, 2019.

Accepted June 18, 2020.

*Corresponding author: mdrake@ncsu.edu ventional milk types were primarily motivated by the belief that organic, local, or pasture-raised milks were superior in sensory quality and congruent with personal values. Overall, QMA was an effective means for verifying conjoint-derived consumer groups and provided a contextual support for conjoint insights. Joint conclusions drawn from the components of this study may serve to guide marketing campaigns and new product development for fluid milk processors.

Key words: milk, focus groups, qualitative multivariate analysis, conjoint

\section{INTRODUCTION}

To counter a decreased interest in fluid milk, it is imperative to understand what features consumers value in fluid milk offerings. However, there has been limited research on consumer motivations and beliefs behind commercial milk preferences and purchase patterns. Instead, the bulk of existing research has been oriented at understanding the effect of intrinsic quality features on consumer approval of fluid milk, such as feed source (Croissant et al., 2007), fat content and appearance (McCarthy et al., 2017a), and off-flavor presence (Hough et al., 2004). To assess these qualities, fluid milk and dairy products have traditionally been judged according to traditional dairy defect standards or evaluated by trained sensory panels. Results from these product assessment methods may then be paired with consumer acceptance data for extrapolating consumer insights from a sensory perspective. Thompson et al. (2004) showed that the combination of analytical and affective sensory techniques via preference mapping was successful in identifying drivers of liking for commercial chocolate milks for different consumer segments. Additionally, combinatorial sensory studies have been performed on unflavored fluid milk to understand drivers of liking, but only for specific populations (Chung, 2009) or for specific processing parameters (Gandy et al., 2008).

Although sensory methods such as defect judging, trained panel profiling, and consumer acceptance test- 
ing are key in predicting consumer preferences, they explain only part of consumer behavior towards commercial fluid milk offerings. Extrinsic product features that do not directly or explicitly affect the sensory qualities of a product also play a large role in consumer purchase and acceptance. Willingness-to-pay and conjoint analysis studies have revealed that milk designated as rBST-free, organic, or local may justify significant price premiums commercially (Schott and Bernard, 2015; Harwood and Drake, 2018). Moreover, the presence of such features may serve to decrease perceived environmental or health risks (DuPuis, 2000), or satisfy alignment with altruistic motivations such as animal welfare (Costanigro et al., 2016; Harwood and Drake, 2018).

Overall, quantitative approaches such as consumer acceptance testing and surveys are exceptional tools for identifying key motivators behind purchase and acceptance. However, the nuanced belief systems and narratives behind those motivations are not easily ascertained with quantitative methods. For this reason, qualitative methods are often used to explain consumer insights from a more holistic context. Past fluid milk studies have primarily relied on focus groups and one-on-one interviews to investigate motivators of acceptance or choice qualitatively. Hill and Lynchehaun (2002) used focus groups to investigate consumer-derived definitions for "organic" and "nonorganic" and found them to be primarily tied to health beliefs, although actual knowledge of organic practices was substantially lacking among respondents.

Probing for gaps in education and finding anecdotal bases for belief systems are among the primary uses of in-person qualitative methods, but collection of such information is inherently at risk of an introduced moderator bias (Caroline Tynan and Drayton, 1988). More recently, a structured approach for collection of qualitative data using assorted methods has been proposed to circumvent concerns of bias. In practice, this set of qualitative methods has been termed qualitative multivariate analysis (QMA), and consists of in-homeusage testing, group discussions, product ranking, and projective mapping (PM; Lopetcharat and Beckley, 2012). The QMA methodology has been used to capture consumer insights for cottage cheese (Drake et al., 2009), cheese shreds (Speight et al., 2019), and pork products (Bittner et al., 2017); however, to our knowledge, QMA has not yet been used for understanding fluid milk consumer insights.

Regardless of what data collection method is used, understanding what product features influence acceptance and purchase patterns can be difficult, as consumer actions are often at odds with stated opinions, beliefs, and preferences (Ulrich and Sarasin, 1995). For this reason, it is important to validate consumer insights by collecting complimentary data sources (qualitative vs. quantitative, implicit vs. explicit) so that congruency can be assessed and inconsistencies can be more aptly explained. Several studies have sought to join quantitative and qualitative data streams for explaining fluid milk consumer desires; however, these insights are generally limited to the combination of sensory analysis (central location test or descriptive analysis) and focus group studies (Porubcan and Vickers, 2005; McCarthy et al., 2017a). Given the ubiquity of internet and computer access, large-scale surveys are increasingly used for garnering quantitative consumer insights. Unfortunately, application of surveys and the resulting collection of big data often fail to consider the diverse individual-level narratives behind the statistics. To bridge the gap between large-scale survey insights and individual-level qualitative insights, this study proposed to cross-validate conjoint-derived consumer segmentation with QMA for commercial fluid milks. Understanding the validity of insights derived from survey data on a qualitative level will provide a richer explanation for consumer purchase behavior, and the congruencies and incongruencies that are uncovered may provide aimed directions for educating and marketing to fluid milk consumers.

\section{MATERIALS AND METHODS}

\section{Experimental Overview}

An adaptive choice-based conjoint (ACBC) analysis survey was conducted to understand consumer preferences for fluid milk offerings. Cluster analysis was subsequently performed on the estimated utility scores from the conjoint survey to understand segmentation among fluid milk consumers. Representative respondents from each identified consumer segment were then invited to participate in a 4-wk QMA study that included 3 wk of home-usage testing, followed by a focus group session in which participants discussed product experiences, ranked the products, and completed a PM exercise. All testing procedures were conducted in compliance with North Carolina State University Institutional Review Board (NCSU IRB) regulations.

\section{Adaptive Choice-Based Conjoint Analysis}

Demographic questions, fluid milk usage questions, and an ACBC survey were developed using Lighthouse Studio (Sawtooth Software version 9.6.1, Orem, UT). Conjoint analysis surveys, including ACBC, conceptually break products down into different features (attributes) and options within those features (levels). 
Randomly compiled product concepts can then be compared within the exercise in a format similar to real-life shopping situations. Repeated product comparisons allow for consumer choice patterns to be investigated, and multivariate analysis techniques can be subsequently applied to identify motivators of choice. Although traditional choice-based conjoint surveys would require analysis of all possible product combinations, the adaptive nature of $\mathrm{ACBC}$ allows for consumer input to refine what options are presented, resulting in lower sample-size requirements and greater survey efficiency (Jervis et al., 2012).

Design and application of the ACBC survey was conducted as described by Harwood and Drake (2018). Briefly, the conjoint consisted of 6 attributes, each with 2 to 5 levels per attribute (Table 1). In addition, analysis of price and price premiums within the ACBC survey was conducted using the summed pricing technique (Cunningham et al., 2010). Several prohibitions were included within the conjoint design to ensure product concepts reflected real marketplace offerings for fluid milk. Prohibitions within the conjoint exercise included the following: rBST-free and organic, rBSTfree and organic pasture-raised, shelf stable, and plastic jug. Participants were instructed before the conjoint exercise that organic milk offerings were, by definition, rBST-free, so the selection of both would be disallowed to ensure no doubling of price premium would be introduced. Following completion of the ACBC survey, respondents were asked if they were willing to participate in a 4-wk in-person study on fluid milk that included in-home-usage testing (wk 1-3), as well as a focus group and PM exercise (wk 4; QMA).

The ACBC survey was uploaded to the North Carolina State University Sensory Service Center database, which consists of over 12,000 members. In all, 719 respondents that self-reported purchase or consumption of fluid milk at least once per month completed the conjoint analysis survey. Participants who completed the entire survey were entered into a drawing to receive one of $15 \$ 25$ gift certificates to a local store.

\section{Qualitative Multivariate Analysis}

To review the validity of segmentation from the conjoint analysis study (which documents consumer perception of product attributes without tangible products), fluid milk consumers from each of the 4 identified consumer segments were invited to participate in the QMA study. Selection for participation in the QMA portion of the study was determined based on root likelihood (RLH) values from the conjoint analysis survey, with respondents who had higher RLH scores receiving higher priority for inclusion. The RLH measurement is akin to goodness of fit for discrete choice data. In all, 20 participants (5 from each segment) were chosen for the study, although only 18 ( $\mathrm{n}=8$ females and 10 males) completed the study. Participants who fully participated in the QMA study were compensated with a $\$ 100$ gift certificate to a local store.

Nine commercial fluid milk products (all half-gallons, 1.5 wk or more from expiration date) were chosen for the QMA study. These products were chosen to capture the breadth of features within the fluid milk market. Selected products included the following: Maple View Farms (locally sourced, HTST), Harris Teeter (store brand, HTST), Harris Teeter Organic (organic, store brand, ultrapasteurized; UP), Harris Teeter UP (store brand, UP), Organic Valley Omega-3 (national brand, organic, DHA-fortified, UP), a2 Milk (national brand, digestibility claim, UP), Fairlife (national brand, UP, ultrafiltered), Horizon Organic (national brand, organic, UP), and Organic Valley Grassmilk (national brand, organic, grass-fed). The home-usage portion of the QMA study was conducted over a 3 -wk period in a randomized complete block design, with each panelist

Table 1. Attributes and levels used in fluid milk conjoint survey

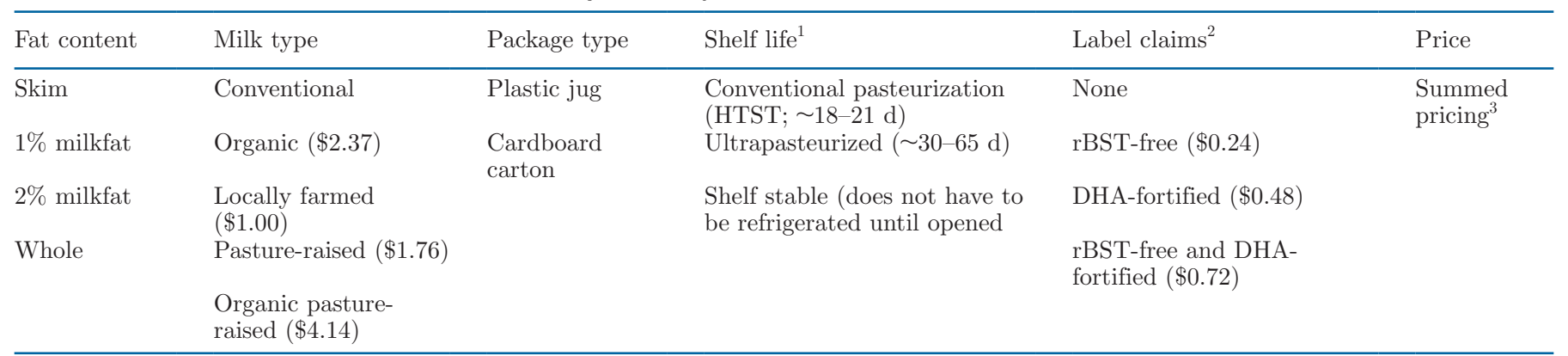

\footnotetext{
${ }^{1}$ Definitions were explained in terms of refrigerated shelf life.

${ }^{2} \mathrm{DHA}=$ docosahexaenoic acid; rBST $=$ recombinant bovine somatotropin.

${ }^{3}$ Summed pricing refers to additive pricing for select product features that carry a price premium. Total summed price ( $\left.\pm 30 \%\right)$ was shown for each assembled product concept in the conjoint exercise.
} 
receiving 3 of the 9 possible milk products per week. Milks for each week of testing were picked up onsite at North Carolina State University and given to panelists in insulated bags with ice packs to ensure product quality and safety. Prior to participation, conjoint utility scores relating to milkfat preferences were evaluated. For participants whose utility scores suggested that they preferred milkfat-containing options $(1 \%, 2 \%$, or whole milk), $2 \%$ milk products were provided throughout the study. For participants who indicated that they preferred skim milk, skim milk products were provided throughout the study, with the exception of the Organic Valley Omega-3 and a2 milk products, where $2 \%$ and $1 \%$ milkfat products were provided, respectively (no skim milk option was offered for these brands). Participants were instructed to try each milk at least once during the week, and were asked to access an online survey (Compusense Cloud, Guelph, ON, Canada) where they rated their overall liking on a 9-point hedonic scale and kept a diary of their likes and dislikes about the product following consumption. Participants were free to use the products in whatever way(s) they chose, so long as those actions mirrored their typical consumption patterns with fluid milk. Once poured or prepared, participants were asked to take a photograph of their chosen usage method and upload the photograph to their online journal entry for the given milk. After completing evaluations, panelists were required to wait a minimum of $10 \mathrm{~h}$ before either re-evaluating a product for a second time (optional), or moving on to their next sample.

Following the 3 wk of home-use evaluation of milks, participants shared their experiences and thoughts about the products during a single 3 -h focus group session. Two focus group sessions ( $\mathrm{n}=9$ each session) were scheduled to accommodate panelist availability and to maintain a reasonable group size for inclusive discussion from all participants. The focus group sessions were video recorded and streamed externally to 2 notetakers to ensure key insights were captured. Prior to beginning the focus groups, consumers were provided with printed records of their home-usage testing diaries, uploaded photos of product use, and summaries of information such as name and price for each product. The first $2 \mathrm{~h}$ of each session were oriented at understanding the values, beliefs, and desires that generally drive the purchase, consumption, and choice of fluid milk products. In addition, each product that was presented in the study was presented by the moderator and thoughts were gathered from each member of the focus group to understand how the aforementioned values and desires were met or unmet by certain products. The group then moved on to a short ranking exercise where they selected their favorite, second favorite, and least favorite of the milks.

Following a brief 15-min break, the session finished with a PM exercise. Panelists were given iPads (Apple Inc., Cupertino, CA) with Compusense Cloud software PM module and were asked to place the products on the map area according to product similarity and dissimilarity. During the PM exercise, panelists were able to review the product packaging, as well as their diary entries and notes from the focus group. Once the products were placed in the map area, panelists had the option of tagging the products with an available list of descriptors. These descriptors included the following terms: low quality, high quality, artificial, natural, safe, expensive, affordable, misleading, sustainable, healthy, and animal welfare-conscious. Similar fluid milk descriptors were suggested to have high consumer relevance in observations reported by Cardoso et al. (2016). A field for free-response descriptors was also included.

\section{Data Analysis}

Responses from the ACBC survey were used to determine individual utility scores, importance scores, and RLH values through the application of hierarchical Bayesian regression (Sawtooth Software version 9.6.1). Respondents with RLH values lower than 0.333 were subsequently removed from further data, as lower RLH values indicate a higher likelihood of "click-through" or random response patterns. To investigate the landscape of consumer opinions regarding fluid milk offerings in more depth, individual utility estimates (18 product feature levels +7 stepwise price levels) from the conjoint survey were subjected to agglomerative hierarchical cluster analysis (rows $=719$ respondents, columns $=25$ total utility levels). Agglomerative hierarchical cluster was performed using a dissimilarity matrix with Euclidean distance proximity type, Ward's agglomeration method, and entropy-based truncation for determination of the number of consumer segments (Cruz et al., 2013). Clustered conjoint data was subsequently subjected to a one-way ANOVA with Fisher's least significant difference posthoc test (95\% confidence).

Data from the PM exercise was exported and organized in a spreadsheet where rows $(\mathrm{n}=9)$ were assigned to each sample and columns $(\mathrm{n}=36)$ were arranged so that $\mathrm{x}$ - and $\mathrm{y}$-coordinates from each panelist's product placement were in sequential columns. In addition, counts of descriptor tag use were calculated for each sample and arranged in a table as supplementary data columns $(\mathrm{n}=11)$. Multiple factor analysis was sub- 
sequently applied to the coordinate data matrix with the descriptor tag counts applied as supplementary variables to construct a biplot that visually explained product differentiation and associated descriptions (XLSTAT version 19.5.2018, Addinsoft, Paris, France).

\section{RESULTS AND DISCUSSIONS}

\section{Conjoint Analysis Survey}

Consumer segments from the conjoint survey were named according to insights gained from their conjoint utility and importance scores. As such, the clusters will be referred to throughout this study as the premium, value, opportunistic, and pragmatic clusters. Premium cluster members were defined by a higher utility for product features with high price premiums and suggestions of enhanced quality, as well as a low care for price. Value cluster members were named as a result of high-price sensitivity relative to other segments. Opportunistic cluster members were defined by a large range of suppressed price sensitivity, and a proclivity for choosing "premium" price features within their price comfort zones. Pragmatic cluster respondents exhibited features similar to both opportunistic and value cluster members, indicating a desire for certain target product features, but an overall adherence to high-price sensitivity. These tendencies will be further explained in the following sections.

Demographically, consumer segments were similar in age and sex proportions, but were more distinct in breakdown of ethnicity, annual household income, and presence of children in the household (Table 2). Specifically, the premium cluster was composed of the highest proportion of non-Caucasian respondents $(44.8 \%)$ and households with children (47.8\%), as well as the lowest proportion of respondents who reported annual household income of $\$ 100,000$ or greater $(22.4 \%)$. These demographics are consistent with previous literature that has documented a heightened likelihood for organic product purchase for non-Caucasian consumers, as well as an unclear link between annual household income and likelihood for organic or premium product purchase (Smith et al., 2009; Harwood and Drake, 2018). The value cluster, on the other hand, was composed of the highest proportion of Caucasian respondents (81.4\%), but was otherwise relatively evenly distributed in terms of income and age. The pragmatic and opportunistic clusters were also relatively evenly distributed demographically, although the pragmatic cluster had the lowest proportion of children in the household (31.4\%) and the opportunistic cluster had the highest proportion of respondents from high-earning households (37.9\% greater than $\$ 100,000 / y r)$.
Observations by Hill and Lynchehaun (2002) indicate that price is of primary importance for fluid milk consumers, even trumping features such as taste and quality. Similarly, price was of primary importance to each consumer segment in the present study; however, significant differences $(P<0.05)$ in price importance were noted among the groups (Figure 1). Specifically, the premium cluster placed the highest importance on milk features unrelated to price, suggesting that this group was most willing to pay price premiums for value-added features. The opportunistic cluster was at parity $(P>$ 0.05 ) with the premium cluster in importance placed on fat content and milk type attributes, but placed a relatively low importance on label claims. The pragmatic cluster was primarily price driven, but did receive an importance score for label claims that was significantly higher $(P<0.05)$ than the opportunistic and value clusters. Finally, the value cluster was lowest overall in allotted importance for attributes unrelated to price, indicating that fluid milk purchase for this group was focused exclusively on product cost.

Ideal builds were assessed based upon utility scores for each segment (Table 3). Some features, such as $2 \%$ milkfat and conventional pasteurization were unanimously preferred among all segments. However, consumer segments showed markedly different ideals for package type, milk type, and label-claim preferences. For these attributes, the premium cluster expressed preference for a fluid milk that was packaged in a plastic jug, organic pasture-raised, and had no label claims. The pragmatic cluster showed similar preferences to the premium cluster, with plastic jug packaging and no label claims; however, organic milk was the preferred milk type for the pragmatic group. The opportunistic cluster also had organic milk type in their ideal build, but additionally showed preference for cardboard carton packaging and rBST-free and DHA label claims. Finally, the value cluster expressed a preference for conventional milk in a plastic jug with no label claims, reinforcing the cluster's general preference for the most basic possible product. Utility for the "none" option, which indicated a group's utility threshold for purchasing a product (if summed utilities of product features from each attribute do not exceed this threshold, the consumer is likely to not choose any option, rather than the options being considered), was also assessed. The value cluster, overall, exhibited the highest $(P<$ $0.05)$ "none" utility, indicating that they were the least likely to be satisfied by a given product offering, and most likely to walk away from a purchase decision if their needs were not met. The premium and pragmatic clusters exhibited a significantly lower "none" utility compared with the value cluster, but significantly higher than the opportunistic cluster, which was lowest 
Table 2. Demographic information for fluid milk consumer clusters $(\mathrm{n}=719)$

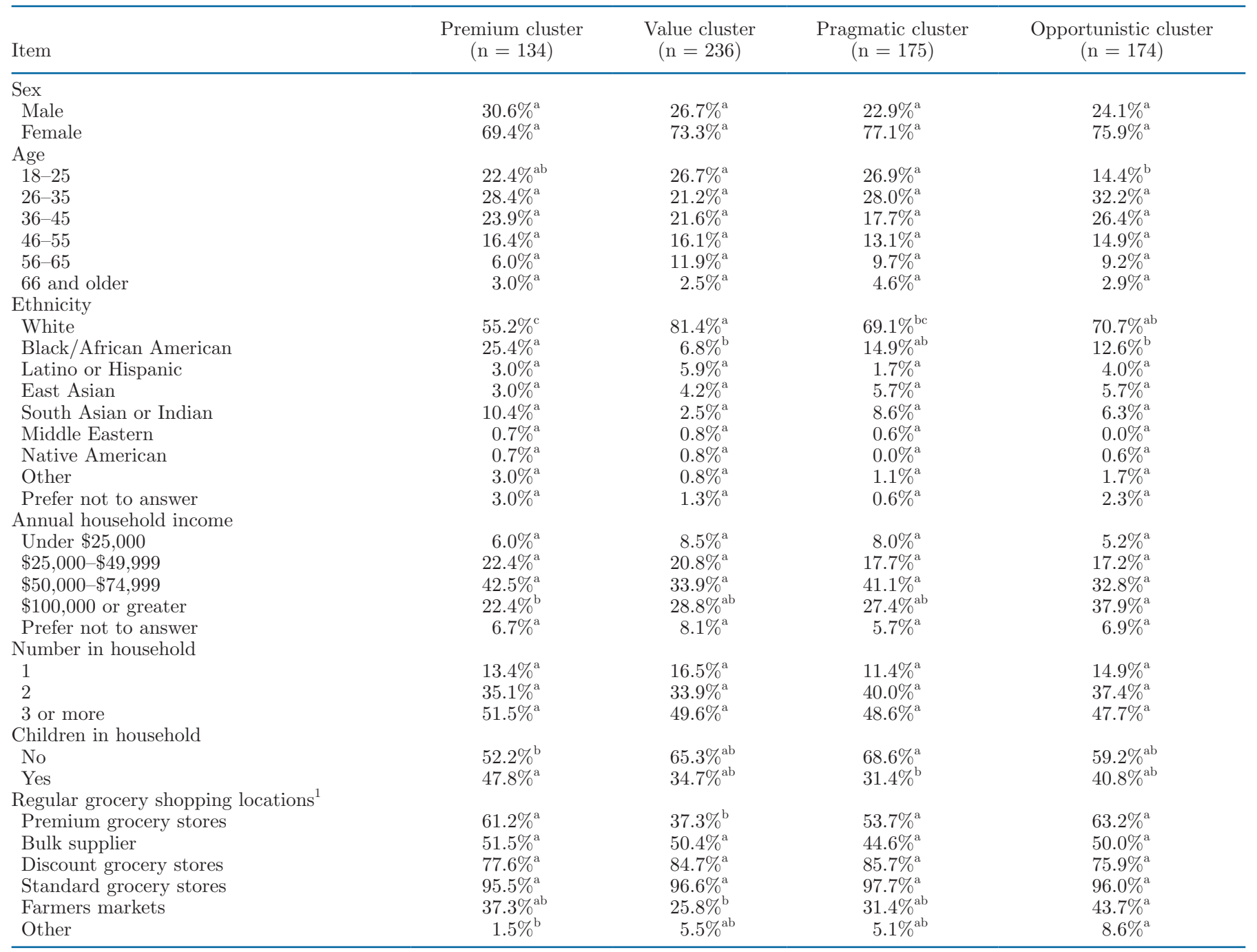

${ }^{a-c}$ Percentages within a row with different superscript letters are significantly different $(P<0.05)$.

${ }^{1}$ Check-all-that-apply question. Totals may exceed $100 \%$.

overall. The relatively low "none" utility for the opportunistic cluster indicated that this group was widely satisfied by various commercial milk offerings, and was more likely to buy a product that doesn't fit their ideals. Investigation into these patterns of preference and price sensitivity were of paramount concern in the subsequent QMA study to better define these concepts within each consumer segment.

\section{QMA Study}

Analysis of journal entries following in-home-usage testing of commercial milks revealed that, overall, consumer opinions on the milks were driven most by price, flavor and aroma, and appearance. In terms of flavor, aroma, and mouthfeel, participants in the study noted several perceived differences among the commercial milks provided. Although participants were unable to attribute these sensory differences to specific product features, previous studies on fluid milk have shown that both trained panelists and consumers are able to detect sensory differences in milk due to different pasteurization techniques (Lee et al., 2017), feed sources (Croissant et al., 2007), and package types (Simon and Hansen, 2001). Secondary features such as packaging type, milk type, nutritional information, shelf life, and functionality as an ingredient were also significant indicators of acceptability for some participants; however, package appearance and label claims (rBST-free, DHAfortified, a2 protein), were more seldom mentioned. Although a variety of product features were mentioned, references to extrinsic product features such as milk 
Table 3. Average utility scores from fluid milk conjoint survey for segmented consumer clusters

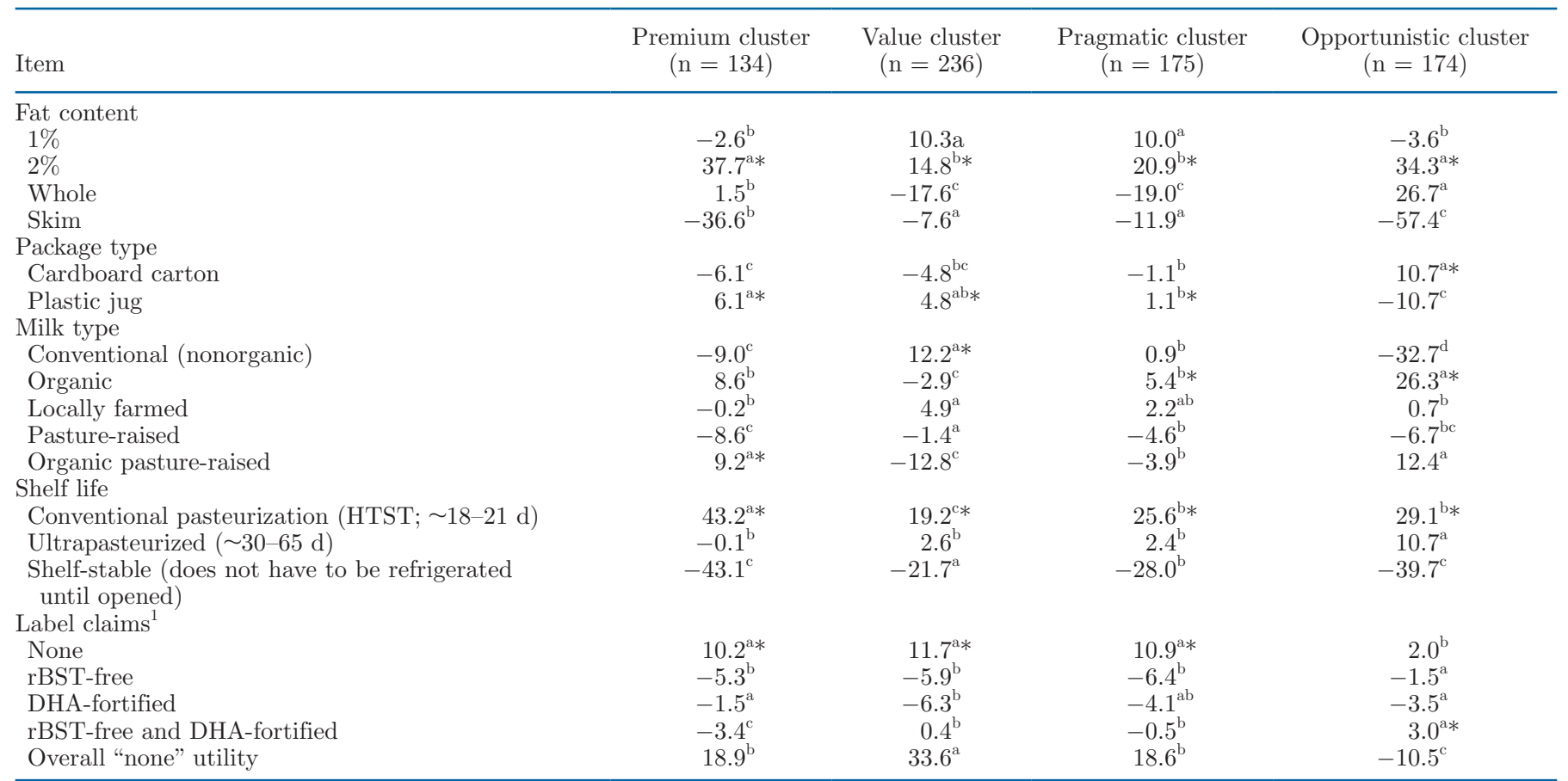

${ }^{\mathrm{a}-\mathrm{c}}$ Means within a row with different superscript letters are significantly different $(P<0.05)$.

${ }^{1} \mathrm{DHA}=$ docosahexaenoic acid; rBST $=$ recombinant bovine somatotropin.

*Indicate ideal build features for that given cluster.

type and label claim were generally discussed in terms of whether the given feature was "worth it" for the price of the product, reinforcing the baseline price sensitivity expressed in the conjoint exercise.
Cluster-related differences were also noted in the post-usage journal entries. Price sensitivity was relatively high for the value, opportunistic, and pragmatic clusters, with references to price or affordability ap-

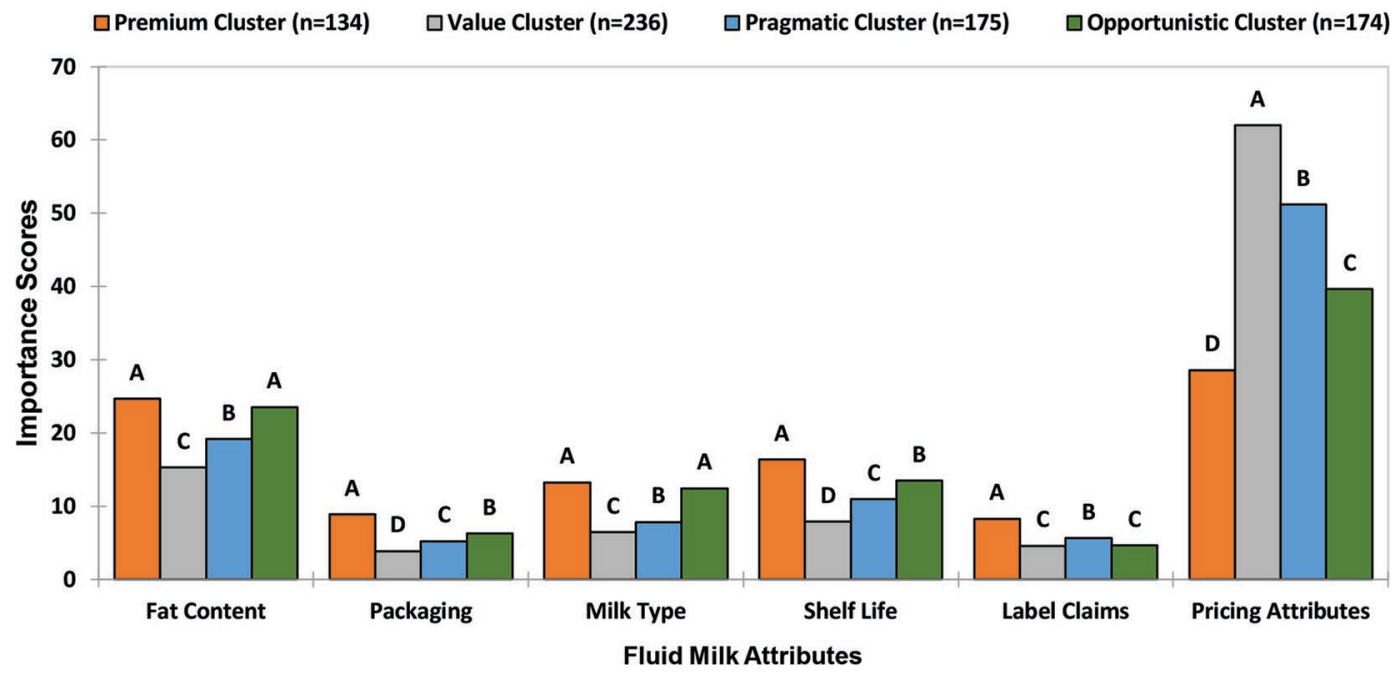

Figure 1. Attribute importance scores from fluid milk conjoint survey for segmented consumer clusters. Letters within attributes (A-D) indicate significant differences between clusters $(P<0.05)$. Sum of importance score values for each cluster is 100 points total, and scores are interpreted as ratio-scaled values. 
pearing in $69.8 \%(88 / 126)$ of journal entries, compared with only $27.8 \%(10 / 36)$ in the premium cluster. This reflected the relatively low importance that the premium cluster exhibited for price in the conjoint study, but suggested that the decreased importance was not simply a function of seeking premium features. Rather, it appeared that price was simply not a feature that was considered as often in the decision-making actions for this group in regard to fluid milk. Furthermore, annual household income and household sizes reported by this group suggested that they have no clear difference in disposable income compared with other groups, further reinforcing an innate difference in approach when assessing commercial milk purchase. Among other differentiating trends found in the journal entries, the pragmatic cluster expressed significantly more concern than the other groups for shelf life and nutritional features. Although no clear inclination for shelf-life importance was noted in the conjoint study, the pragmatic cluster did place significantly more importance on label claims than the opportunistic or value clusters, which may show that this group is more likely to read and explore product information that is readily available on the package to ensure they make an informed purchase. In this way, attention to shelf life by pragmatic cluster-type consumers may be driven by a desire to use the product features they sought out without fear of spoilage.

Photo uploads from the QMA diaries revealed that commercial milks were consumed in a variety of ways. Primarily, the milks were consumed (in order of frequency) in the following ways: as-is, with cereal, as a coffee creamer, as a meal ingredient, and as a base for shakes or smoothies. Preference for usage style was relatively consistent throughout the study, with most consumers opting to consume all 9 milks in the same fashion. The pragmatic and opportunistic clusters were the least likely to consume the milks on their own, choosing instead to use the milks as functional ingredients (e.g., frothed for coffee) or flavor compliments (e.g., a base for smoothie). In a review of milk proteins, Fox (2001) noted that adult populations primarily consume milk as an ingredient and, as such, may derive value from milk's functional properties. As noted in the conjoint study, the opportunistic and pragmatic clusters were defined by choice patterns that seemingly keyed in on specific product features. These choice patterns, coupled with differences in propensity to review product information and consume milk products in a variety of fashions, implied that purchase behaviors may be governed by seeking features they feel imply quality or functionality for a given use. Furthermore, the opportunistic cluster exhibited a relatively high utility for ultrapasteurization, although conventional pasteuriza- tion was their ideal option. Even though this result may simply reflect a lack of knowledge of commercial organic milk production (typically ultrapasteurized), it may also suggest that opportunistic consumers seek a product with a longer shelf life because their uses for milk are outside of simply drinking it as a beverage.

Focus groups with QMA participants were oriented at more thoroughly understanding consumer experiences with the tested commercial milks, as well assessing the value of various product features. Furthermore, these opinions were used to add context to the analysis of the PM exercise and home-usage test results. Visually, product spaces from the PM exercise indicated that there was clear differentiation of the commercial milks (Figure 2). Panelists tended to differentiate the milks based on perception of quality and affordability on factor 1 (56.01\% variability explained) and based on trust factors (misleading, artificial, safe, natural) on factor 2 (21.58\% variability explained). Nonorganic store brand products (Harris Teeter, HTST and UP) were generally associated with being affordable, but having low quality. The concept of private label milks being perceived as lower quality has been well-documented in previous studies, although price and store image may significantly affect these perceptions of private label grocery products (Richardson et al., 1994; Hovhannisyan and Gould, 2012). Liking scores (not pictured) for these products indicated that the Harris Teeter UP product was among the bottom 3 milks in liking for each group except the value cluster, for which it was tied for the highest liking score among the milks evaluated.

Milks such as a2 milk, Horizon Organic, and Organic Valley Grassmilk were associated with being expensive and high quality, as well as healthy, sustainable, animal welfare-conscious, safe, and natural. Overall, each of these milks received relatively high liking scores from each consumer group, with no milk receiving a bottom 3 score in liking; however, the highest liking scores came from participants in the pragmatic and opportunistic clusters. Specifically, the pragmatic cluster gave its highest liking scores to Horizon Organic and a2 milk. Preference for a national brand organic product reflected conjoint preferences for the pragmatic group, whereas the acceptance for a2 milk highlighted the group's aforementioned tendency to review product details and make an informed choice to meet their needs. McCarthy et al. (2017b) showed that both milk and plant-based milk alternative consumers value products that promise digestive benefits. Although no clear within-cluster trend was determined during the focus groups, many participants expressed interest in the a2 product and its claims for better digestibility, further suggesting that this may be a motivating feature for milk consumers. The opportunistic cluster expressed a 


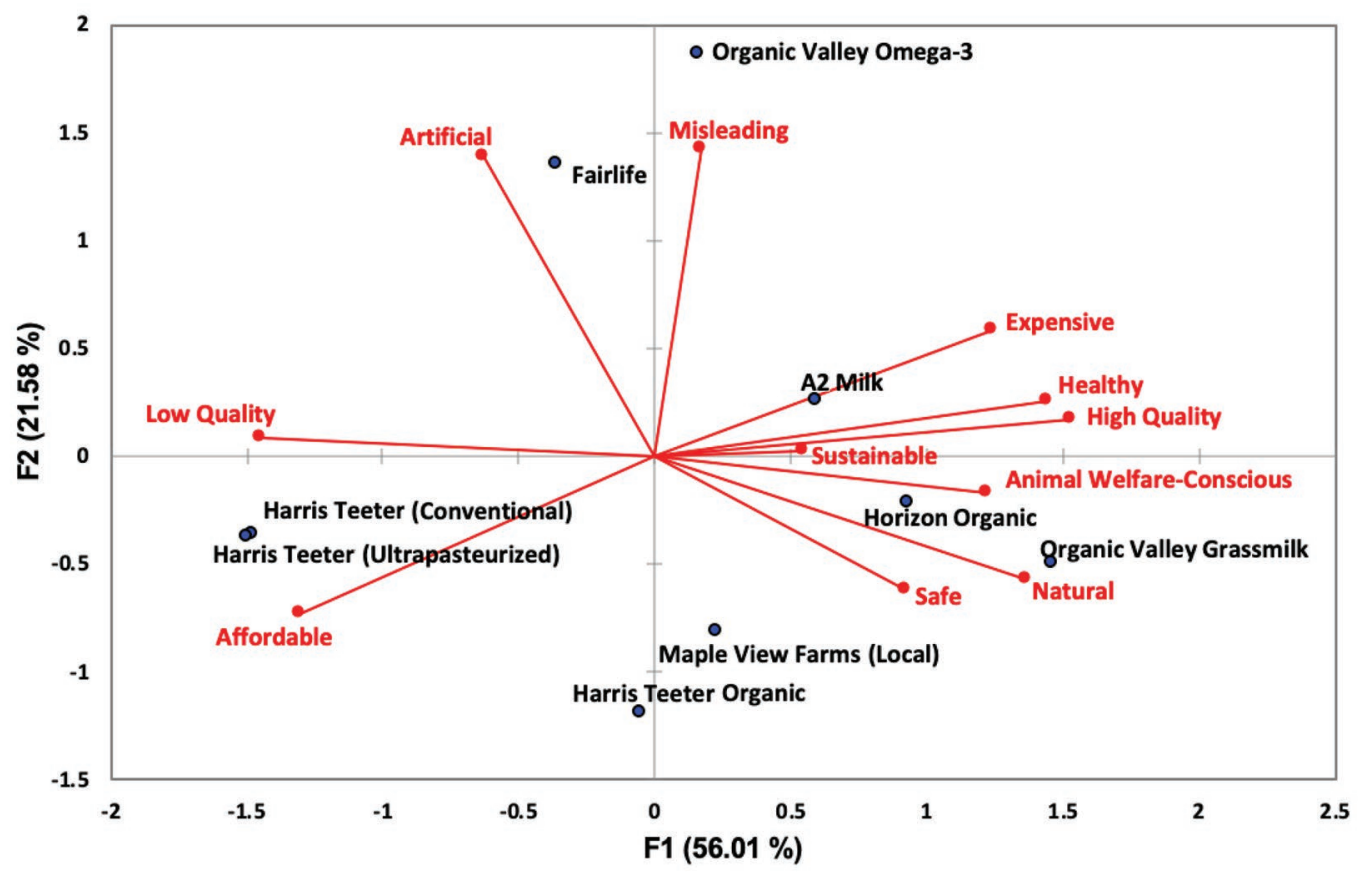

Figure 2. Projective map of commercial brand fluid milks and descriptive product features from qualitative multivariate analysis study participants $(\mathrm{n}=18) . \mathrm{F} 1=$ factor 1 variability explained; $\mathrm{F} 2=$ factor 2 variability explained.

similarly high opinion for the Horizon Organic product, scoring it as their second most-liked sample, which mirrored conjoint preferences for a product that was organic and packaged in a cardboard carton. Organic Valley Grassmilk was the most liked sample for the opportunistic cluster, overall. The most preferred milk type option in the conjoint study was organic for the opportunistic cluster, though they received the highest utility score $(P<0.05)$ for the organic pasture-raised milk type among all clusters. This suggested that consumers within this segment were characterized by a baseline desire for organic designation in their commercial milk choice and are willing to explore additional "premium" features or claims if the total product price is within their price comfort range. Analysis of price utility scores for the opportunistic cluster suggested that, compared with other groups, they had a relatively large price range they would consider for commercial milks. Most groups exhibited a proportional decrease in utility with increase in price, but the opportunistic group showed stable utility scores until a total price of approximately $\$ 5.00$ was reached, after which utility steeply dropped (Figure 3 ). Given the $\$ 5.00$ price point coincides with the price threshold of several "premium" commercial milk offerings, it was unsurprising that the opportunistic cluster was defined by preference for these items, but willing to explore more basic products if the premium products are too expensive.
Along factor 2, the descriptors "artificial" and "misleading" loaded positively, and were strongly associated with the Fairlife and Organic Valley DHA milk samples. Focus group insights indicated that many consumers believed these samples to be "artificial" for different reasons. For the Fairlife sample, several participants pointed out that the removal of lactose and difference in protein content as a result of ultrafiltration made the product, by definition, artificial in nature, although many also reported these features as an added value. Furthermore, many noted in the focus group and in their journals that the Fairlife sample was markedly different in aroma and mouthfeel compared with the other milks. Overall, both the Fairlife and Organic Valley DHA samples received polarized liking scores among the consumer clusters. The pragmatic cluster gave a higher score to Fairlife than any other group, indicating that high protein content or lactose-free might be motivating features for a subset of fluid milk consumers. Similarly, the Organic Valley DHA milk was liked strongly by the premium cluster and, surprisingly, the value cluster, whereas it was least liked by the pragmatic cluster and second-least liked for the opportunistic cluster.

In contrast to the Fairlife and Organic Valley DHA milks, the Harris Teeter Organic and Maple View Farms (Hillsborough, NC) milks were negatively correlated with "artificial" and "misleading" descriptors. 


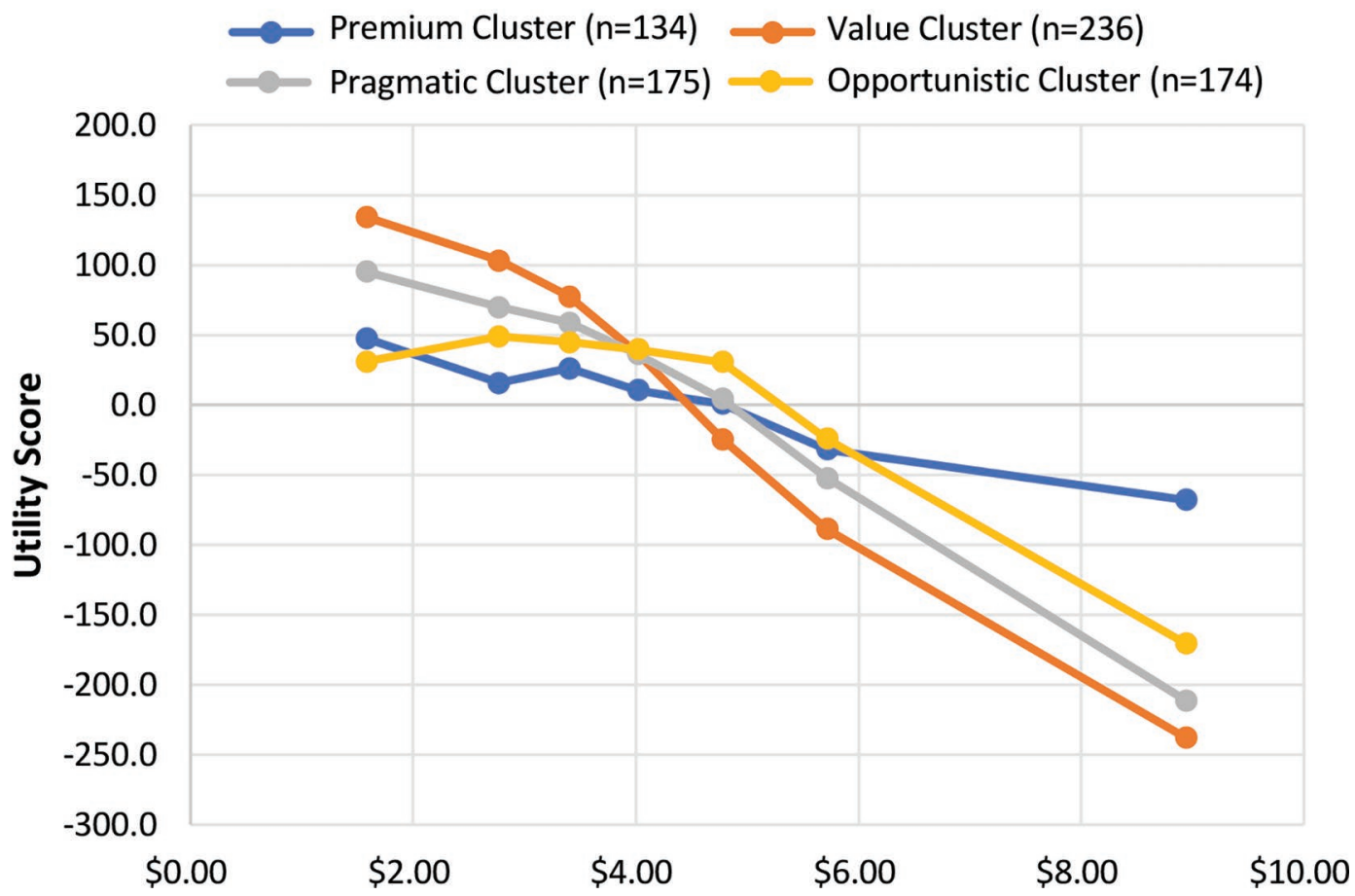

Figure 3. Conjoint-derived price sensitivity of fluid milk consumer clusters.

Additionally, these products did not load along factor 1 , indicating that these samples were, overall, believed to be intermediate in terms of affordability and quality. The Harris Teeter Organic product did not elicit strong reactions from any group, generally scoring toward the middle for each (between 3rd and 6th). Journal responses and focus group notes echoed the implications of these intermediate liking scores, with more price-sensitive panelists (value, opportunistic, pragmatic cluster members) indicating that price was too high, and others commenting that the milk was "no real standout" (pragmatic cluster member) and that many other milks were "better than this one" (premium cluster member). The Harris Teeter Organic product was also particularly criticized in journal entries as having a strange flavor and aftertaste, which several focus group participants attributed to ultrapasteurization. Decreased liking as a result of cooked flavors associated with ultrapasteurization have been widely noted in previous milk studies (Gandy et al., 2008; Lee et al., 2017), although there was no clear indication of why other ultrapasteurized samples in the study were not penalized. On the other hand, the Maple View Farms (local) milk was more polarizing among the consumer groups.

The locally-farmed milk (Maple View Farms) was not very appealing for members of the pragmatic and opportunistic clusters (rated 7th and 4th, respectively); however, it particularly appealed to premium and value cluster consumers, scoring first for each group. Premium cluster members spoke highly of the milk quality (flavor, aroma, mouthfeel), as well as the local designation, which was a perceived as a value-added feature compared with other milks in the study. In addition, premium cluster members reported that the glass container "made [the milk] seem fresher" and induced a nostalgic feeling that represented "the old way milk was bought," corroborating focus group insights on glass milk packaging reported by Hollywood et al. (2013). Value cluster members found the Maple View Farms milk appealing for many of the same reasons as the premium cluster: sensory quality, attractive container, and local designation. In the conjoint exercise, the value cluster expressed a preference for conventional milk, likely due to the lower price; however, locally-farmed milk type designation was the next most preferred milk type for the group, and was the highest average utility for locally farmed among all groups. In the case of Maple View Farms, value cluster members also reported that the milk was a desirable option because the milk had a bottle-buy-back program that would credit them $\$ 1.50$ for returning the bottle to their grocer. In this way, the value cluster effectively mirrored their conjoint-derived preference for local designation, while maintaining their relatively strict price standards. Other QMA participants mentioned that the bottle deposit system was a 
feature they were unlikely to use, thereby leaving their perception of price unchanged for this product. Observations by Grimes-Casey et al. (2007) suggest that milk consumers are, overall, more likely to participate in deposit-refund programs for milk if the deposit is high, although their initial purchase likelihood decreases proportionally to the increased deposit price. Considering the draw that local designation and glass packaging presented for some fluid milk consumers, investigation of ideal deposit-return pricing models that work for both consumers and retailers may be warranted.

\section{CONCLUSIONS}

Cross-validation of conjoint analysis and QMA studies revealed that survey-derived consumer sentiments for fluid milk largely agreed with qualitative measures such as home-usage testing and focus groups. Generally, this suggests that rapid testing procedures, such as conjoint analysis, are able to accurately predict consumer segmentation for commodity products such as fluid milk. As indicated in the conjoint study, price sensitivity was the largest determinant of consumer segmentation, although certain consumer groups did value other "premium" product features. Beyond price, consumer clusters in the study were defined by their unique preferences for milk type, packaging, and the presence or absence of other value-added features. Specifically, the premium cluster exhibited preferences for nonconventional milk types that implied higher quality. The value cluster valued the most basic available product features, but showed a willingness to explore locally-farmed milks. The pragmatic cluster allotted relatively little importance to anything but price, but decisively preferred organic milk to other available milk types, including pasture-raised and organic pastureraised, which are often conflated with organic designation. Finally, the opportunistic cluster expressed acceptability for a larger group of product options compared with other groups, but generally preferred organic milk packaged in cardboard or paperboard cartons with added features such as DHA-fortification or rBST-free label claims. The application of QMA showed that these preferences generally play out in real-life interactions with commercial milk offerings, and may additionally be influenced by factors such as household size, personal beliefs, convenience, and proclivity to read product information and labeling. Additionally, QMA insights and application of PM suggested that consumers implicitly relate nonconventional milk types with higher quality. To this end, investigation into the presence of implicit biases related to milk production and milk type designation is a needed area of future research. Furthermore, QMA revealed that consumers perceive differences in flavor, aroma, and mouthfeel among commercial milk products. Although these features may not be readily captured using online survey techniques such as conjoint, they may help expand on consumer likelihood of repeat purchases, assuming the conceptual needs required for initial purchase are met. Understanding of consumer insights for fluid milk from a macro survey perspective supported by anecdotal qualitative insights is extremely valuable for developing targeted marketing strategies, and it is integral for future growth within the fluid milk market.

\section{ACKNOWLEDGMENTS}

This study was funded in part by The Dairy Alliance (Atlanta, GA) and the National Dairy Council (Rosemont, IL). The use of trade names does not imply endorsement or lack of endorsement of those not mentioned. The authors have not stated any conflicts of interest.

\section{REFERENCES}

Bittner, E. P., H. Ashman, M. Hastie, R. J. van Barneveld, A. H. Hearn, N. Thomson, and F. R. Dunshea. 2017. Innovation in an expanding market: Australian pork is not a commodity. Anim. Prod. Sci. 57:2339-2344. https://doi.org/10.1071/AN17299.

Cardoso, C. S., M. J. Hötzel, D. M. Weary, J. A. Robbins, and M. A. von Keyserlingk. 2016. Imagining the ideal dairy farm. J. Dairy Sci. 99:1663-1671. https://doi.org/10.3168/jds.2015-9925.

Caroline Tynan, A., and J. L. Drayton . 1988. Conducting focus groups-A guide for first-time users. Mark. Intell. Plann. 6:5-9. https://doi.org/10.1108/eb045757.

Chung, S. J. 2009. Effects of milk type and consumer factors on the acceptance of milk among Korean female consumers. J. Food Sci. 74:S286-S295. https://doi.org/10.1111/j.1750-3841.2009.01224.x.

Costanigro, M., O. Deselnicu, and D. T. McFadden. 2016. Product differentiation via corporate social responsibility: Consumer priorities and the mediating role of food labels. Agric. Human Values 33:597-609. https://doi.org/10.1007/s10460-015-9640-9.

Croissant, A. E., S. P. Washburn, L. L. Dean, and M. A. Drake. 2007. Chemical properties and consumer perception of fluid milk from conventional and pasture-based production systems. J. Dairy Sci. 90:4942-4953. https://doi.org/10.3168/jds.2007-0456.

Cruz, A. G., R. S. Cadena, W. F. Castro, E. A. Esmerino, J. B. Rodrigues, L. Gaze, J. A. F. Faria, M. Q. Freitas, R. Deliza, and H. M. A. Bolini. 2013. Consumer perception of probiotic yogurt: Performance of check all that apply (CATA), projective mapping, sorting and intensity scale. Food Res. Int. 54:601-610. https://doi .org/10.1016/j.foodres.2013.07.056.

Cunningham, C. E., K. Deal, and Y. Chen. 2010. Adaptive choicebased conjoint analysis: A new patient-centered approach to the assessment of health service preferences. Patient 3:257-273. https: //doi.org/10.2165/11537870-000000000-00000.

Drake, S. L., K. Lopetcharat, and M. A. Drake. 2009. Comparison of two methods to explore consumer preferences for cottage cheese. J. Dairy Sci. 92:5883-5897. https://doi.org/10.3168/jds.2009-2389.

DuPuis, E. M. 2000. Not in my body: BGH and the rise of organic milk. Agric. Human Values 17:285-295. https://doi.org/10.1023/ A:1007604704026.

Fox, P. F. 2001. Milk proteins as food ingredients. Int. J. Dairy Technol. 54:41-55. https://doi.org/10.1046/j.1471-0307.2001.00014.x.

Gandy, A. L., M. W. Schilling, P. C. Coggins, C. H. White, Y. Yoon, and V. V. Kamadia. 2008. The effect of pasteurization tempera- 
ture on consumer acceptability, sensory characteristics, volatile compound composition, and shelf-life of fluid milk. J. Dairy Sci. 91:1769-1777. https://doi.org/10.3168/jds.2007-0833.

Grimes-Casey, H. G., T. P. Seager, T. L. Theis, and S. E. Powers. 2007. A game theory framework for cooperative management of refillable and disposable bottle lifecycles. J. Clean. Prod. 15:1618 1627. https://doi.org/10.1016/j.jclepro.2006.08.007.

Harwood, W. S., and M. A. Drake. 2018. Identification and characterization of fluid milk consumer groups. J. Dairy Sci. 101:8860-8874. https://doi.org/10.3168/jds.2018-14855.

Hill, H., and F. Lynchehaun. 2002. Organic milk: Attitudes and consumption patterns. Br. Food J. 104:526-542. https://doi.org/10 .1108/00070700210434570.

Hollywood, L., L. Wells, G. Armstrong, and H. Farley. 2013. Thinking outside the carton: Attitudes towards milk packaging. Br. Food J. 115:899-912. https://doi.org/10.1108/BFJ-Jul-2010-0127.

Hough, G., L. Garitta, and R. Sánchez. 2004. Determination of consumer acceptance limits to sensory defects using survival analysis. Food Qual. Prefer. 15:729-734. https://doi.org/10.1016/j.foodqual 2004.02.006

Hovhannisyan, V., and B. W. Gould. 2012. A structural model of the analysis of retail market power: The case of fluid milk. Am. J. Agric. Econ. 94:67-79. https://doi.org/10.1093/ajae/aar124.

Jervis, S. M., J. M. Ennis, and M. A. Drake. 2012. A comparison of adaptive choice-based conjoint and choice-based conjoint to determine key choice attributes of sour cream with limited sample size. J. Sens. Stud. 27:451-462.

Lee, A. P., D. M. Barbano, and M. A. Drake. 2017. The influence of ultra-pasteurization by indirect heating versus direct steam injection on skim and $2 \%$ fat milks. J. Dairy Sci. 100:1688-1701. https: //doi.org/10.3168/jds.2016-11899.

Lopetcharat, K., and J. Beckley. 2012. Qualitative multivariate analysis. Pages 100-121 in Product Innovation Toolbox: A Field Guide to Consumer Understanding and Research. John Wiley \& Sons, Oxford, UK.

McCarthy, K. S., K. Lopetcharat, and M. A. Drake. 2017a. Milk fat threshold determination and the effect of milk fat content on consumer preference for fluid milk. J. Dairy Sci. 100:1702-1711. https: //doi.org/10.3168/jds.2016-11417.
McCarthy, K. S., M. Parker, A. Ameerally, S. L. Drake, and M. A. Drake. 2017b. Drivers of choice for fluid milk versus plant-based alternatives: What are consumer perceptions of fluid milk? J. Dairy Sci. 100:6125-6138. https://doi.org/10.3168/jds.2016-12519.

Porubcan, A. R., and Z. M. Vickers. 2005. Characterizing milk aftertaste: The effects of salivation rate, PROP taster status, or smal changes in acidity, fat, or sucrose on acceptability of milk to milk dislikers. Food Qual. Prefer. 16:608-620. https://doi.org/10.1016/ j.foodqual.2005.01.007.

Richardson, P. S., A. S. Dick, and A. K. Jain. 1994. Extrinsic and intrinsic cue effects on perceptions of store brand quality. J. Mark. 58:28-36. https://doi.org/10.1177/002224299405800403.

Schott, L., and J. Bernard. 2015. Comparing consumer's willingness to pay for conventional, non-certified organic and organic milk from small and large farms. J. Food Distrib. Res. 46:186-205.

Simon, M., and A. P. Hansen. 2001. Effect of various dairy packaging materials on the shelf life and flavor of pasteurized milk. J. Dairy Sci. 84:767-773. https://doi.org/10.3168/jds.S0022-0302(01)74532 $-8$.

Smith, T., B. H. Lin, and C. Huang. 2009. Growth and development in the US retail organic food sector. Sustainability 1:573-591. https: //doi.org/10.3390/su1030573.

Speight, K. C., A. N. Schiano, W. S. Harwood, and M. A. Drake 2019. Consumer insights on prepackaged Cheddar cheese shreds using focus groups, conjoint analysis, and qualitative multivariate analysis. J. Dairy Sci. 102:6971-6986. https://doi.org/10.3168/jds .2018-16209.

Thompson, J. L., M. A. Drake, K. Lopetcharat, and M. D. Yates. 2004. Preference mapping of commercial chocolate milks. J. Food Sci. 69:S406-S413. https://doi.org/10.1111/j.1365-2621.2004 .tb09958.x.

Ulrich, P. C., and C. Sarasin. 1995. Facing Public Interest: The Ethical Challenge to Business Policy and Corporate Communications. Kluwer Academic Publications, London, UK.

\section{ORCIDS}

M. A. Drake @ https://orcid.org/0000-0002-4744-2493 